Kansas State University Libraries

New Prairie Press

\title{
UNREPLICATED VARIETY TRIALS: EFFECTS OF CHECK PLOT DENSITY AND FIXED VERSUS RANDOM TREATMENTS
}

\author{
Tisha Maas \\ Jeanette Stafford \\ Boi Sebolai \\ David Marx \\ Daryl Travnicek
}

See next page for additional authors

Follow this and additional works at: https://newprairiepress.org/agstatconference

Part of the Agriculture Commons, and the Applied Statistics Commons

\section{(c) (1) $\Theta(9$}

This work is licensed under a Creative Commons Attribution-Noncommercial-No Derivative Works 4.0 License.

\section{Recommended Citation}

Maas, Tisha; Stafford, Jeanette; Sebolai, Boi; Marx, David; Travnicek, Daryl; and Pedersen, Jeff (2003). "UNREPLICATED VARIETY TRIALS: EFFECTS OF CHECK PLOT DENSITY AND FIXED VERSUS RANDOM TREATMENTS," Conference on Applied Statistics in Agriculture. https://doi.org/10.4148/2475-7772.1184

This is brought to you for free and open access by the Conferences at New Prairie Press. It has been accepted for inclusion in Conference on Applied Statistics in Agriculture by an authorized administrator of New Prairie Press. For more information, please contact cads@k-state.edu. 
Author Information

Tisha Maas, Jeanette Stafford, Boi Sebolai, David Marx, Daryl Travnicek, and Jeff Pedersen 


\title{
Unreplicated Variety Trials: Effects of Check Plot Density and Fixed Versus Random Treatments
}

\author{
Tisha Maas, Jeanette Stafford, Boi Sebolai, Dr. David Marx, and Dr. Daryl Travnicek, \\ Department of Biometry \\ Dr. Jeff Pedersen, USDA-Agricultural Research Service \\ University of Nebraska-Lincoln \\ Lincoln, NE 68583-0712
}

\begin{abstract}
Crop researchers performing germplasm screenings are often unable to replicate their plots due to scarcity of seed and the large numbers of genotypes being evaluated. The use of known check varieties is a common method of overcoming the difficulties associated with unreplicated trials. In this simulation, we explored the effect of check plot density on the effectiveness of the resulting analysis. We also explored the effect of analyzing treatments as random versus fixed. Our study considers ten different designs with check densities ranging from $5 \%$ of the plots to $50 \%$. The designs and analyses were then compared on the basis of the correlation of the actual treatment effects with the following: "observed" yield, LSMEANs for treatments fixed, and BLUPs for treatments random. Finally, we observed the frequency with which the analysis ranked the top $10 \%$ of the treatments within the top $15 \%$ of the LSMEANs or BLUPs. It was found that the LSMEANs and BLUPs from the spatial analysis provide more accurate results than the observed Y-values. Also, if the treatments are analyzed as fixed and the LSMEANs are used as estimates, then there seems to be a certain point beyond which not much additional information is gained by adding more check plots. This plateau is reached near a check plot density of approximately $30 \%$. Finally, the BLUPs seem to be a more accurate estimate of the true treatment effects than are the LSMEANs at the lower densities; in fact, the BLUPs perform relatively well even at check densities of only $5 \%$ or $10 \%$.
\end{abstract}

\section{INTRODUCTION}

Quantifying and adjusting for variability due to the spatial structure of a field is an essential step in field variety trials. Estimation of spatial structure is made more difficult when the experimental varieties are not replicated. In these cases, there is no "internal" method of accounting for spatial structure. To help alleviate this problem, a "check" variety is often planted in a systematic pattern throughout the field. The check variety is then used to determine the spatial structure of the field so that the data from the experimental varieties can be adjusted and thus evaluated more accurately.

When considering the use of a check variety for a field study, several problems confront the researcher. Among these are the pattern in which to plant the checks, the proportion of 
checks to put in the field, and whether to analyze the experimental varieties as fixed or random treatments. The first of these is mentioned briefly by Burgueño et. al. (2000) and is explored in some detail by Maas (2002). The latter two issues are the focus of this study. To address these, we ran simulations based on a 10 by 10 (100 plot) grid and considered ten different designs with check densities ranging from $5 \%$ of the plots to $50 \%$.

\section{METHODS}

\section{Check Pattern Selection}

The patterns of checks were chosen based on the ideas mentioned by Maas (2002) relating to a principle of maximum separation of check plots. For the lower densities, our check patterns attempted to minimize the distance from each experimental variety to its nearest check. For the higher densities where all of the experimental varieties are adjacent to at least one check, we used the distance to the second closest check, then third closest, etc. In conjunction with these distance criteria, we also examined the number of checks that were a given distance from each experimental variety. Our aim was to maximize the number of checks that were closest to the experimental varieties and to minimize the number that were farthest away. All ten designs are shown in Figure 1 (a through $\mathrm{j}$ ).

\section{Data Generation}

Treatment numbers were assigned to each of the 100 plots. Check plots were assigned a treatment number of " 0 ", and non-check plots were each assigned a unique positive value. We began by simulating a spatial floor on the 100 plots, and we used the spherical semivariogram to create this spatial floor. The equation for the spherical semivariogram is as follows:

$$
\gamma\left(d_{i j}\right)=\left\{\begin{array}{l}
\sigma^{2}\left\{1.5\left(d_{i j} / \rho\right)-.5\left(d_{i j} / \rho\right)^{3}\right\} \text { if } \mathrm{d}_{\mathrm{ij}} \leq \rho \\
\sigma^{2} \text { otherwise }
\end{array}\right.
$$

where $d_{i j}$ is the distance between observation $i$ and observation $j$. The sill of the semivariogram is denoted by $\sigma^{2}$, and $\rho$ is the range. For simplicity we chose a no-nugget structure. Our sill was set at 1.0 for the entire simulation. Recall that our 100 plots form a $10 \times 10$ grid. We wanted to evaluate the performance of each density when only varieties close together were correlated, as well as when the spatial correlation spanned the entire field. Thus, we chose "small" and "large" ranges of $\rho=3.5$ and $\rho=15$, respectively. When we analyze the data with treatments fixed, our model equation is as follows:

$y_{i j}=\mu+\tau_{i}+\varepsilon_{i j}$, with

$\operatorname{Var}\left(\varepsilon_{i j}\right)=\sigma^{2} f\left(d_{i j}\right)$ where $f\left(d_{i j}\right)=\left\{\begin{array}{l}1-1.5\left(d_{i j} / \rho\right)+.5\left(d_{i j} / \rho\right)^{3} \text { if } \mathrm{d}_{\mathrm{ij}} \leq \rho \\ 0 \text { otherwise }\end{array}\right.$ 
When we analyze the treatments as random, our model equation is given as

$$
\begin{aligned}
& y_{i j}=\mu+\tau_{i}+\varepsilon_{i j}, \text { with } \\
& \operatorname{Var}\left(\tau_{i}\right)=\sigma^{2} \tau \\
& \operatorname{Var}\left(\varepsilon_{i j}\right)=\sigma^{2} f\left(d_{i j}\right) .
\end{aligned}
$$

We used the RANNOR generator (with $\sigma^{2}{ }_{\tau}=0.25$ ) to simulate these different treatment effects. We then added these random treatment effects onto the existing spatial floor of the non-check plots. Without loss of generality, no treatment effect was added to plots containing check varieties. Our final simulated data set contained a variable for the treatment effect only and another for the combined spatial floor and treatment effect (Y). The ' $\mathrm{Y}$ ' variable represents "observed" field data. Note that in practice, a researcher does not know if the treatments are random or fixed. Thus, even though the data has been generated with random treatment effects, we analyzed it with both treatments fixed and treatments random to see how the results differed.

\section{$\underline{\text { Analysis }}$}

For each range/density combination, we generated and analyzed 1,000 data sets. Initially, we analyzed the simulated data sets with only the information from the check plots to estimate the spatial structure, using the following PROC MIXED statements:

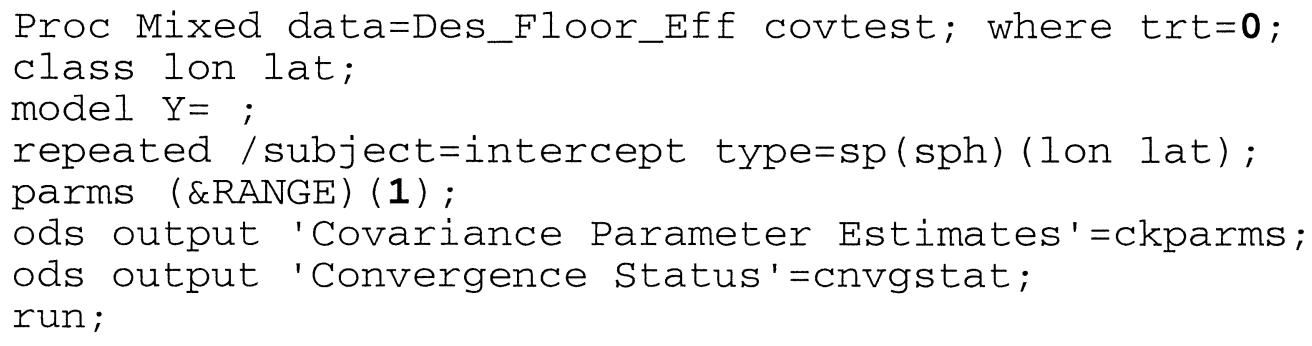

We assigned a convergence code (for use in the final analysis) to each iteration based on the 'Convergence Status' and the 'Covariance Parameter Estimates'. We have found that PROC MIXED can sometimes converge to unrealistic estimates for the range and sill when it is used to analyze spatial experiments. In this simulation, we considered any estimate that was smaller than $10^{-6}$ or larger than 100 to be unfeasible. If the analysis converged to reasonable estimates of the range and sill, we assigned a code of " 1 ". The covariance parameter estimates themselves were then output to a dataset to be used in the next stage of the analysis. On the other hand, if the analysis converged, but the parameters were obviously inappropriate (as described above), we assigned a code of " 2 ". Finally, a code of " 3 " was assigned if the analysis failed to converge. Then, based on the convergence code, the data from each iteration was subsequently analyzed as follows. 
If the iteration was assigned a code of " 1 ", meaning that the initial analysis converged to logical parameters, then we used a spherical spatial structure to analyze the full dataset that included the experimental varieties. First, we analyzed the treatments as fixed (SAS code given below). Note the NOITER option in the PARMS statement: the range and sill were held fixed at the values given by the initial analysis using only checks.

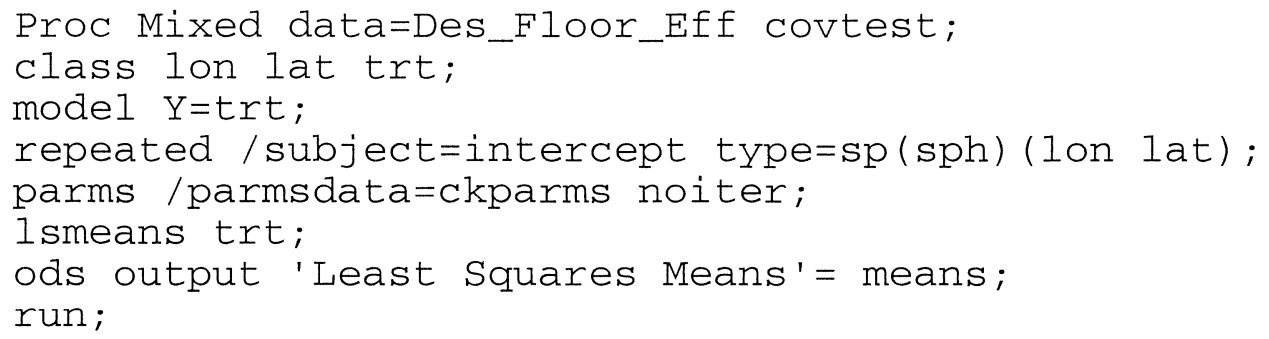

Next, we analyzed the treatments as random (SAS code given below). When treatments were analyzed as random, the data set containing initial values for the parameters also contained a variable for the treatment variance. In this analysis, the treatment variance was allowed to iterate, whereas the range and sill were held fixed. Also, since we have simulated a positive treatment variance, the LOWERBOUND option was used to keep the treatment variance away from zero.

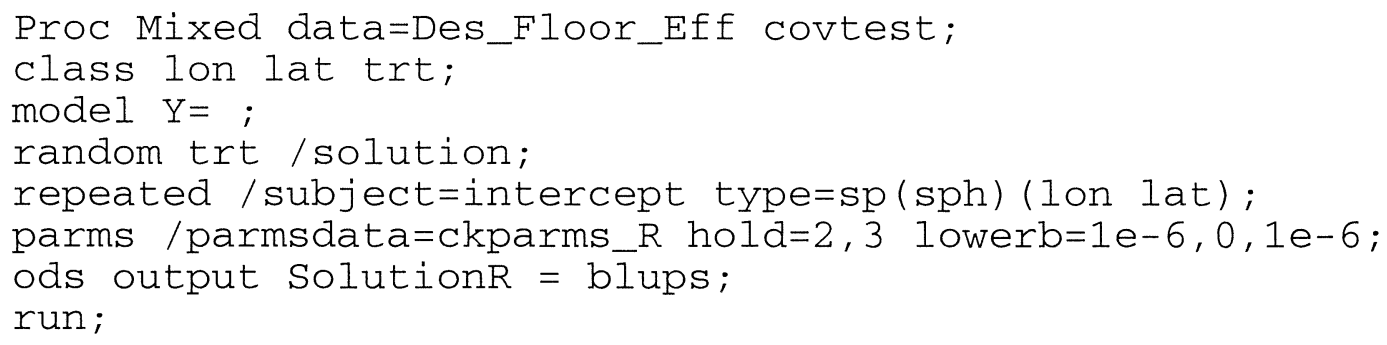

If an iteration was coded as a " 2 " or a " 3 ", then we were left with no reasonable values for the range and sill. Note that if a linear semivariogram is used to adjust for spatial variability, then the choice of parameters does not affect the estimates. That is, the LSMEANS and BLUPS will not depend on the intercept or slope of the semivariogram (Marx 1987). Thus, we chose to use a linear structure if the initial analysis did not provide us with feasible estimates of the range and sill. Parameters of 0.01 and 100 were used in the PARMS statement, resulting in a slope of 1 and a range large enough to encompass the entire field (PROC MIXED uses the reciprocal of the range in the linear structure, hence 0.01). First we analyzed the treatments as fixed (SAS code shown below). Note that the range and sill were held fixed.

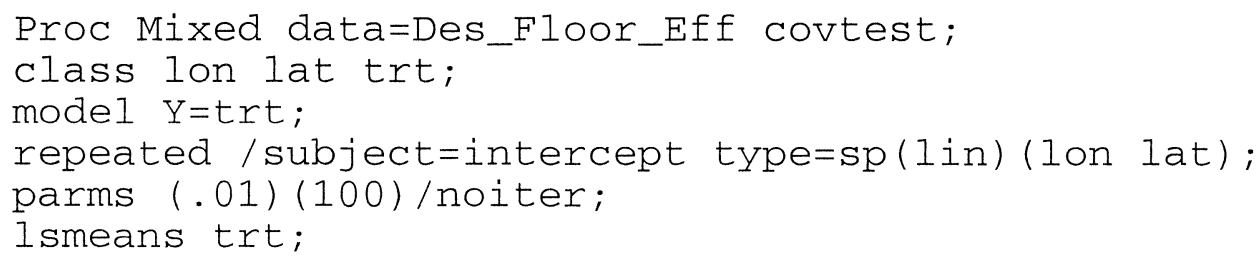


ods output 'Least Squares Means'= means;

run;

Next, we analyzed the treatments as random (SAS code below). Again, note that the range and sill were held fixed, while the treatment variance was allowed to iterate. Note that in the PARMS statement, the initial value for the treatment variance was given as .5 even though our simulated treatment variance was .25. As mentioned earlier, our intent was to keep the treatment variance away from zero; thus, we started the treatment variance on the other side of .25 with the same purpose in mind.

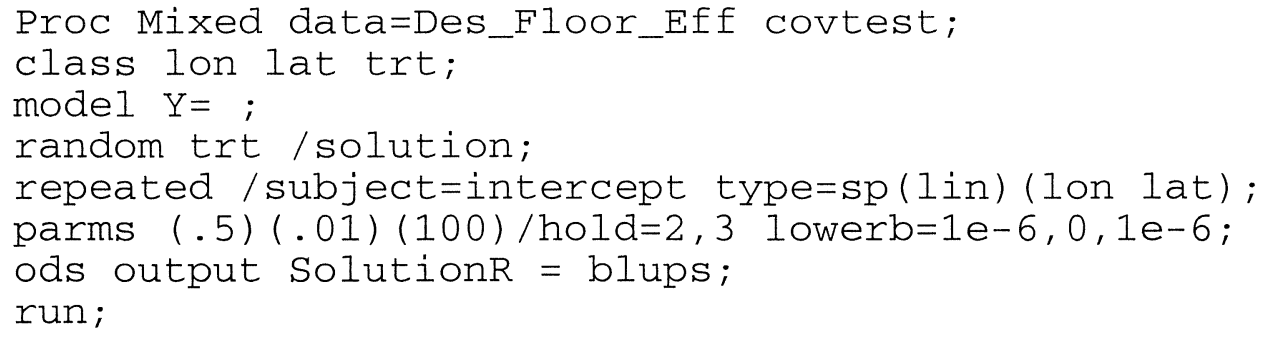

\section{Output Data Sets}

From each iteration (regardless of whether the iteration was coded as a "1", "2", or " 3 "), we output the LSMEAN, BLUP, the simulated treatment effect, and the "observed" Y-value for each of the observations. Then we compiled a data set consisting of the LSMEANs, BLUPs, simulated treatment effects, and the Y-values from all iterations. One such data set was generated for each density/range combination. Then, we calculated the correlation of the actual treatment effects with each of the following: "observed" yield, LSMEANs, and BLUPs. Note that the LSMEANs and BLUPs are simply adjusted versions of the Y-values. Since the LSMEANs and BLUPs have adjusted for the underlying spatial variation, we would expect them to be a more accurate representation of the actual treatment effects; thus, we expect the LSMEANs and BLUPs to be more highly correlated with the treatment effects than the "observed" Y-values. Finally, in addition to the correlations, we also analyzed the ranks of the LSMEANs, BLUPs, and Y-values in relation to the simulated treatment effects. In large variety field trials, the researcher is primarily interested in finding the top experimental varieties and then conducting further studies using only those varieties. With that in mind, we observed the frequency with which the analysis ranked the top $10 \%$ of the treatments within the top $15 \%$ of the LSMEANs, BLUPs, or Y-values. We define this as percent success. For example, in the case of $30 \%$ check density, there are 70 experimental varieties in our field to which we assigned treatment effects. The top ten percent of those 70 varieties are those ranked from 1 to 7 . If the LSMEAN for one of those varieties was ranked into the top fifteen percent of the LSMEANs, then that was defined as a success (note that in determining the top ten percent the researcher considers only numerical differences; i.e., the differences are not necessarily significant). This was repeated for the BLUPs and Y-values, and for each density. Again, since the LSMEANs and BLUPs have adjusted for spatial variability, we expect them to do a better job of identifying what truly are the top treatments as compared to the Y-values. 


\section{RESULTS}

Figure 2 ( $a$ and $b$ ) shows the correlations of the LSMEANs, BLUPs, and Y-values with the simulated treatment effects for each of the different densities. Here, we highlight some of the basic patterns that appear. As expected, we see that the correlation of the Y-values with the treatment effects remains constant as the density varies. Also, we see that the correlation of the LSMEANs with the treatment effects is relatively low for the smaller densities and increases as the density increases; i.e., as we gain information regarding the underlying spatial variability, the LSMEAN becomes a more accurate representation of the true treatment effect. Furthermore, we see that the LSMEANs seem to reach somewhat of a plateau around 30-35\% for the smaller range and around $20-25 \%$ for the larger range. Finally, we see that the correlations of the BLUPs with the treatment effects are relatively high, regardless of the check pattern density.

One interesting feature appears in Figure 2(a). Notice that at the 5\% density, the Yvalues are more correlated with the treatment effect than are the LSMEANs when the range is small. This is due to the check pattern itself. Upon examination of the 5\% density pattern, one can see that none of the checks are within 3.5 units of each other. Thus, when the range is 3.5 , we do not gain any information and thus cannot make adjustments to our estimates regarding the underlying spatial variability.

Next, Figure 3 ( $a$ and $b$ ) shows plots of the percent success over all iterations for the short and long range, respectively. As with the correlation plots, the same basic patterns emerge. The Y-values are consistently low for both ranges, the LSMEANs begin low and increase to a plateau (which is more visible for the longer range), and the BLUPs perform consistently better than either the Y-values or the LSMEANs, especially at the lowest densities.

In the plot for the short range, Figure 3(a), another feature stands out. For the LSMEANs and BLUPs, the patterns of increase are not as smooth as in the correlation plots. Between the $25 \%$ and $45 \%$ densities there is a spike and dip pattern. This pattern is still present, although much less pronounced, in the long range plot (Figure 3 (b)). It may be that the $25 \%$ and $45 \%$ patterns are more optimal than we thought, or that the $30 \%$ and $35 \%$ are less optimal than expected at estimating the short range. For the long range, all densities are performing closer to where we expected them.

\section{DISCUSSION AND CONCLUSIONS}

First of all, the LSMEANs and BLUPs from the spatial analysis provide more accurate results than the observed Y-values. Also, if the treatments are analyzed as fixed and the LSMEANs are used as estimates, then there seems to be a certain point beyond which not much additional information is gained by adding more check plots. If the range is small, this plateau is reached when approximately $30-35 \%$ of the plots contain the check variety; however, when the range is large, this plateau is reached a bit sooner (when $20-25 \%$ of the plots contain checks). This information may be very important to the researcher who is deciding how to allocate 
resources. There is a balance point between the literal cost of having more checks in the field versus the cost of information lost by not having enough checks, and finding this balance point is key to a researcher who is designing an unreplicated experiment. Finally, the BLUPs seem to be a more accurate estimate of the true treatment effects than are the LSMEANs at the lower densities; in fact, the BLUPs perform relatively well even at check densities of only $5 \%$ or $10 \%$.

Another interesting problem arose from this simulation study which we would like to highlight. Recall that when treatments were analyzed as random, we used the lowerbound option to keep the treatment variance away from zero. This led to some surprising results! Whether we used a lower bound of $10^{-6}$ or $10^{-8}$ (i.e., anything close but not equal to zero), if the treatment variance reached that lower bound and stayed at a value close to it, then we saw an interesting effect. The correlations of the BLUPs with the treatment effects remained relatively high and the ranks were unchanged, even though the BLUPs themselves were very closely centered on the exact same value.

In conclusion, it seems that the check plot density does have an effect on the efficiency of the resulting analysis. As the proportion of checks in the field increases, so does the amount of information we gain. This is true up to a certain point, as mentioned above. It seems that when around $30 \%$ of the plots contain checks, the information we would gain by adding more checks may not be worth the sacrifice of space in the field. Also, this study indicates that BLUPS are a more accurate representation of the treatment effects. However, the authors feel that additional research is necessary to confirm this finding, and we hesitate to state that treatments should be analyzed as random. 


\section{References Cited}

Burgueño, J., A. Cadena, J. Crossa, M. Bänziger, A. R. Gilmour, and B. Cullis. 2000. User's Guide for Spatial Analysis of Field Variety Trials Using ASREML. Mexico, DF: CIMMYT.

Maas, Tisha and David B. Marx. 2002. "Unreplicated Spatial Designs Compared Using Optimality Criteria." Proceedings of the 2002 Kansas State University Conference on Applied Statistics in Agriculture. Manhattan, KS: Kansas State University: pp. 84-93.

Marx, David B. and Kevin C. Thompson. 1987. Practical Aspects of Agricultural Kriging. Division of Agriculture, University of Arkansas. Bulletin No. 903.

\section{Additional References}

Cullis, Brian, Bev Gogel, Arūnas Verbyla, and Robin Thompson. 1998. "Spatial Analysis of Multi-Environment Early Generation Variety Trials." Biometrics 54, pp. 1-18.

Marx, David B. 1992. "Designed Experiments in the Presence of Spatial Correlation." Proceedings of the 1992 Kansas State University Conference on Applied Statistics in Agriculture. Manhattan, KS: Kansas State University: pp. 104-124.

Marx, David B. and Walter W. Stroup. 1993. "Analysis of Spatial Variability Using PROC MIXED." Proceedings of the 1993 Kansas State University Conference on Applied Statistics in Agriculture. Manhattan, KS: Kansas State University: pp. 40-59.

SAS Institute. 2002. SAS OnlineDoc, Version 8.2. Cary, NC: SAS Institute, Inc. 
Figure 1.

a) $5 \%$

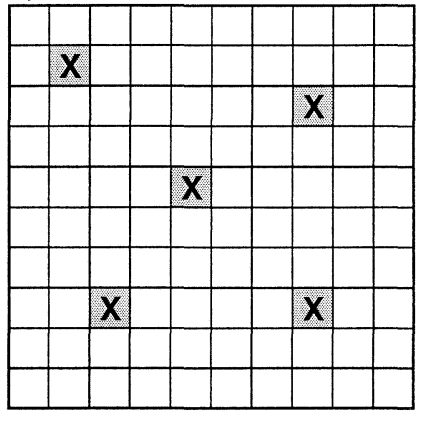

d) $20 \%$

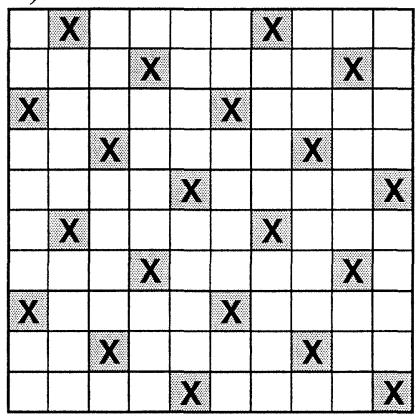

g) $35 \%$

\begin{tabular}{|c|c|c|c|c|c|c|c|}
\hline & & & $\bar{X}$ & & $\bar{X}$ & & \\
\hline & & $x$ & & $x$ & & $\bar{x}$ & \\
\hline & $\underline{x}$ & & $\underline{x}$ & & & $x$ & \\
\hline $\mathbf{X}$ & & $x$ & & $x$ & & $\bar{x}$ & \\
\hline & & & $\bar{x}$ & & $\mathbf{X}$ & & \\
\hline $\mathbf{x}$ & & $\mathrm{X}$ & & $\bar{x}$ & & $\bar{x}$ & \\
\hline & $\underline{x}$ & & 2 & & & $\mathrm{X}$ & \\
\hline $\mathbf{X}$ & & $x$ & & $\mathrm{X}$ & & $\underline{X}$ & \\
\hline & 6 & & $\bar{x}$ & & $\bar{X}$ & & \\
\hline $\mathbf{X}$ & & $\mathbf{X}$ & & $x$ & & $\underline{\mathbf{x}}$ & \\
\hline
\end{tabular}

j) $50 \%$

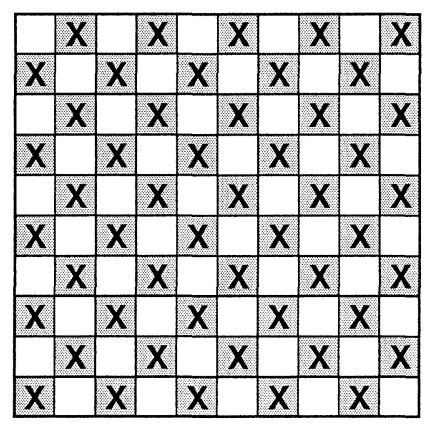

b) $10 \%$

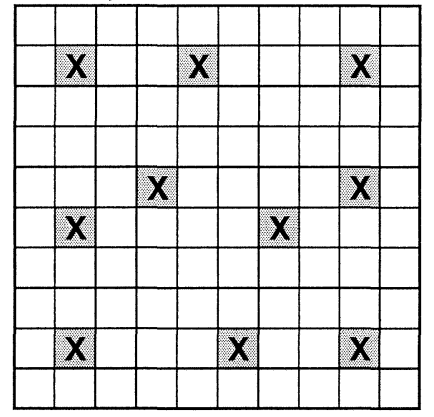

e) $25 \%$

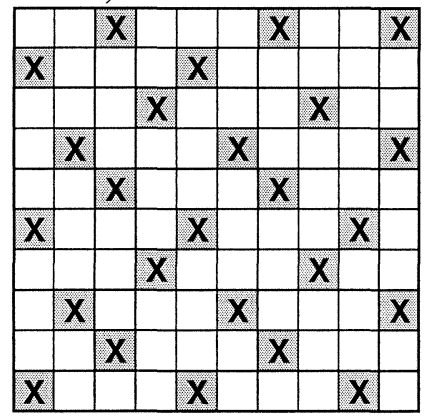

h) $40 \%$

\begin{tabular}{|c|c|c|c|c|c|c|c|c|}
\hline & & & & & & X & & $\bar{x}$ \\
\hline $\mathbf{X}$ & 7 & & & ת & & & & \\
\hline & $\mathbf{n}$ & & $\lambda$ & & $\bar{X}$ & & & $\bar{x}$ \\
\hline $\mathbf{X}$ & & 7 & & $\bar{x}$ & & & $\bar{x}$ & \\
\hline 2 & $\underline{x}$ & & $x$ & & & $\mathbf{x}$ & & $x$ \\
\hline $\mathbf{X}$ & 2 & & & $\mathbf{x}$ & & & & \\
\hline & $\underline{x}$ & & $\mathrm{x}$ & & $\bar{x}$ & & & $x$ \\
\hline $\bar{X}$ & & 2 & & $\bar{x}$ & & & & \\
\hline & & & $\bar{x}$ & & & $x$ & & $x$ \\
\hline$X$ & & & & $\mathrm{X}$ & & & & \\
\hline
\end{tabular}

c) $15 \%$

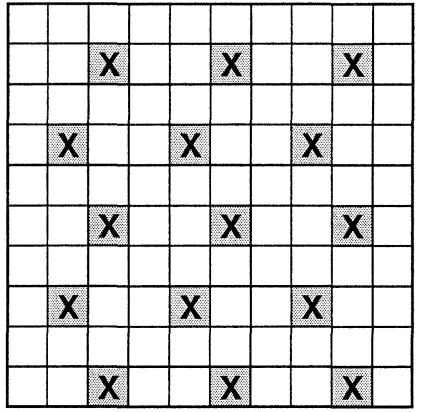

f) $30 \%$

\begin{tabular}{|c|c|c|c|c|c|}
\hline & $\mathrm{X}$ & & $\bar{X}$ & & $x$ \\
\hline $\mathbf{x}$ & & $x$ & & $\bar{x}$ & \\
\hline & $\mathbf{x}$ & & $\mathrm{X}$ & & $\mathbf{x}$ \\
\hline $\mathbf{X}$ & & $x$ & & $\bar{x}$ & \\
\hline & $\mathrm{x}$ & & $\bar{x}$ & & $\mathbf{x}$ \\
\hline$x$ & & $x$ & & $x$ & \\
\hline & $\bar{x}$ & & $\mathbf{x}$ & & $\mathbf{x}$ \\
\hline $\mathrm{X}$ & & $x$ & & $\bar{x}$ & \\
\hline & $\bar{x}$ & & $\underline{x}$ & & $x$ \\
\hline $\bar{X}$ & & $\mathbf{X}$ & & $\bar{x}$ & \\
\hline
\end{tabular}

i) $45 \%$

\begin{tabular}{|c|c|c|c|c|c|c|c|c|}
\hline & $\bar{x}$ & 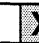 & & & $\bar{x}$ & & $\underline{\bar{x}}$ & \\
\hline $\mathbf{x}$ & & $x$ & & $\bar{x}$ & & $\mathbf{X}$ & 2 & $\bar{x}$ \\
\hline & $x$ & & & & $\bar{X}$ & & $\bar{x}$ & \\
\hline$x$ & & $\underline{x}$ & $x$ & & & $\underline{x}$ & 2 & \\
\hline & $\underline{x}$ & & & & $x$ & & $\underline{x}$ & \\
\hline $\mathbf{x}$ & & $\bar{K}$ & & $x$ & & $\mathbf{X}$ & $\gamma$ & \\
\hline & $\bar{x}$ & & & & $\bar{x}$ & & $x$ & \\
\hline$x$ & & $\underline{x}$ & $\underline{x}$ & & & $\bar{x}$ & $\underline{2}$ & \\
\hline & $\bar{x}$ & & & & $\mathbf{x}$ & & $\bar{x}$ & \\
\hline $\bar{x}$ & & $\underline{x}$ & & $\bar{x}$ & & $\mathbf{X}$ & & $\mathbf{X}$ \\
\hline
\end{tabular}


Figure 2. Correlations With Treatment Effects.

(a) Range $=3.5$

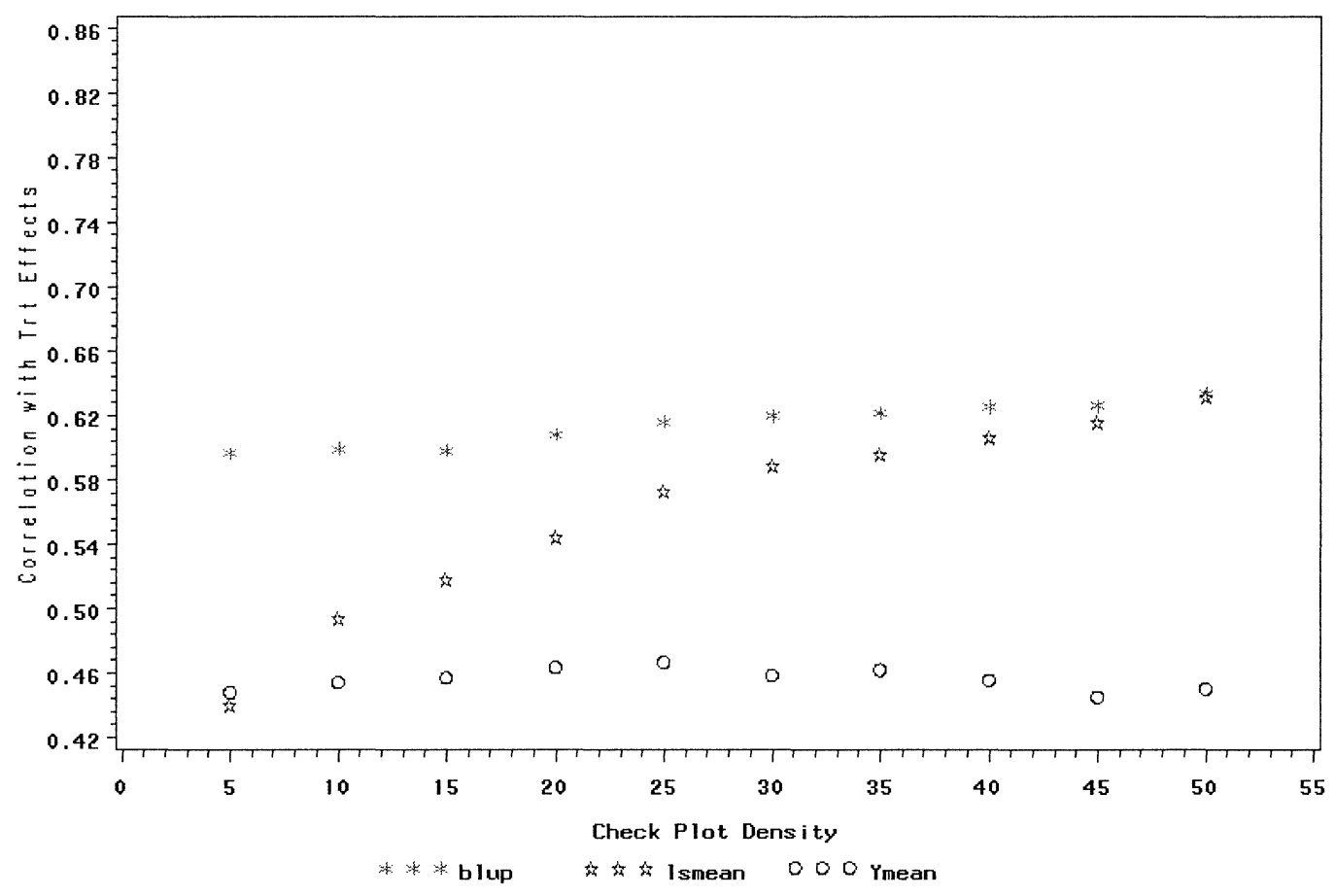

(b) Range $=15$

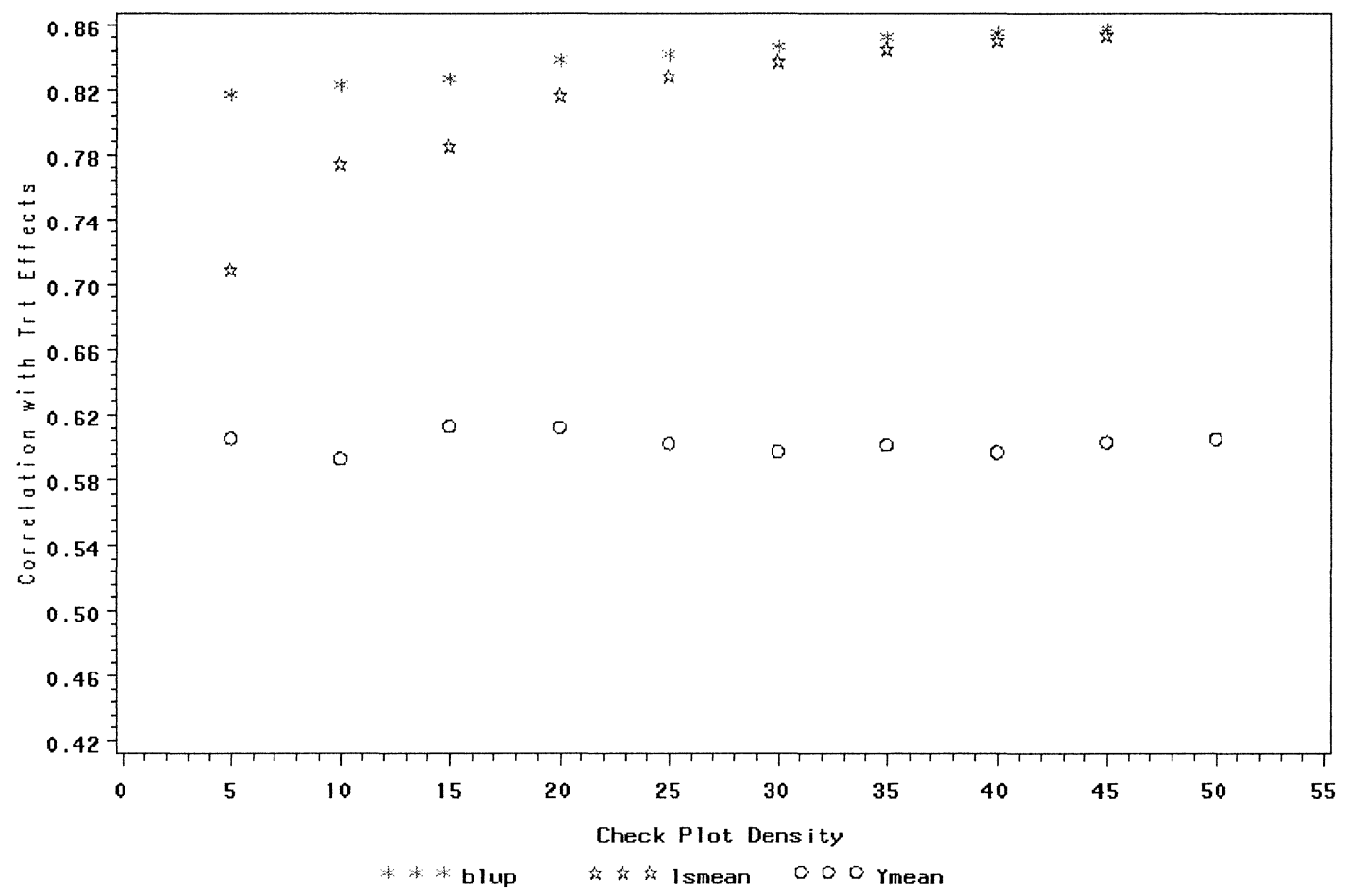


Figure 3. The frequency with which the analysis ranked the top $10 \%$ of the treatments within the top $15 \%$ of the LSMEANS, BLUPS, or Y-values.

(a) Range $=3.5$

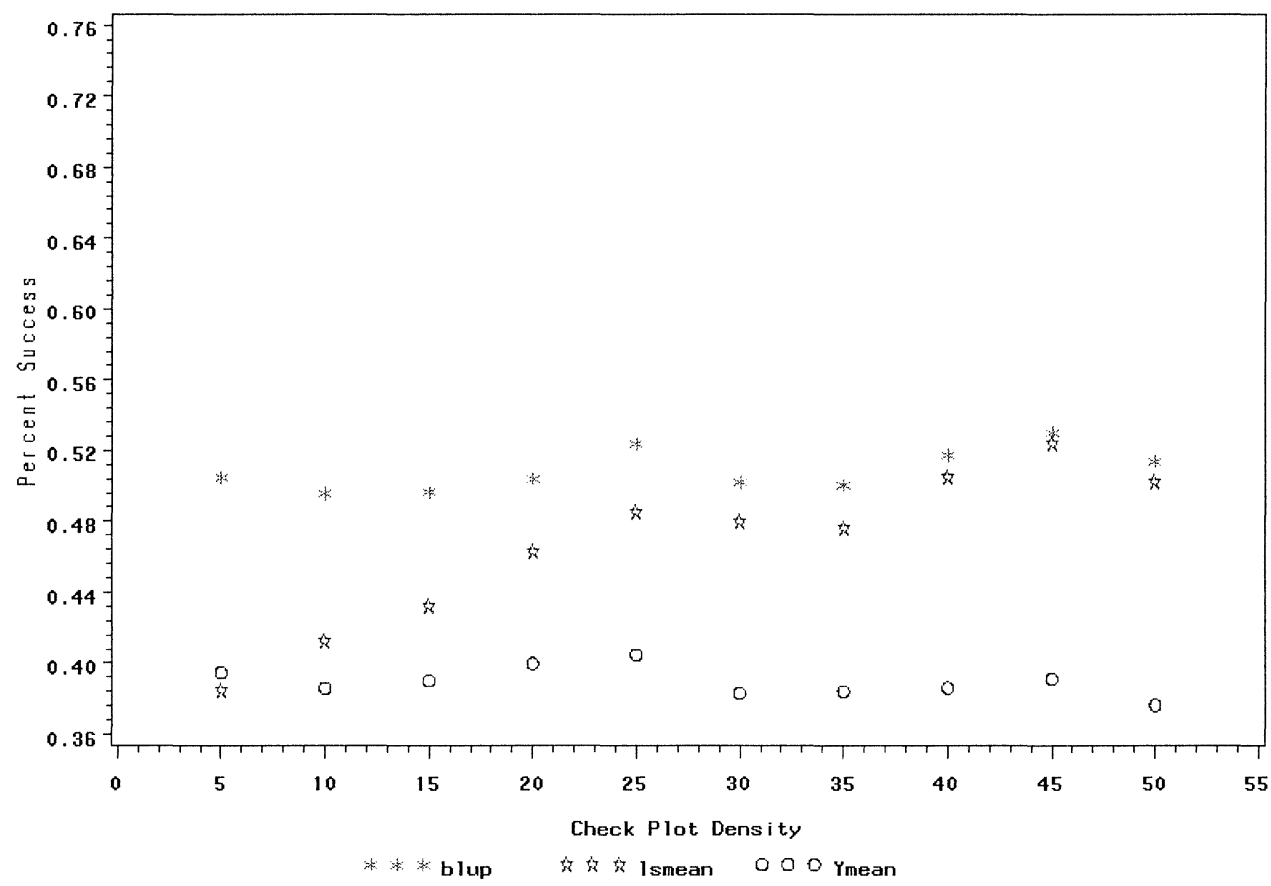

(b) Range $=15$

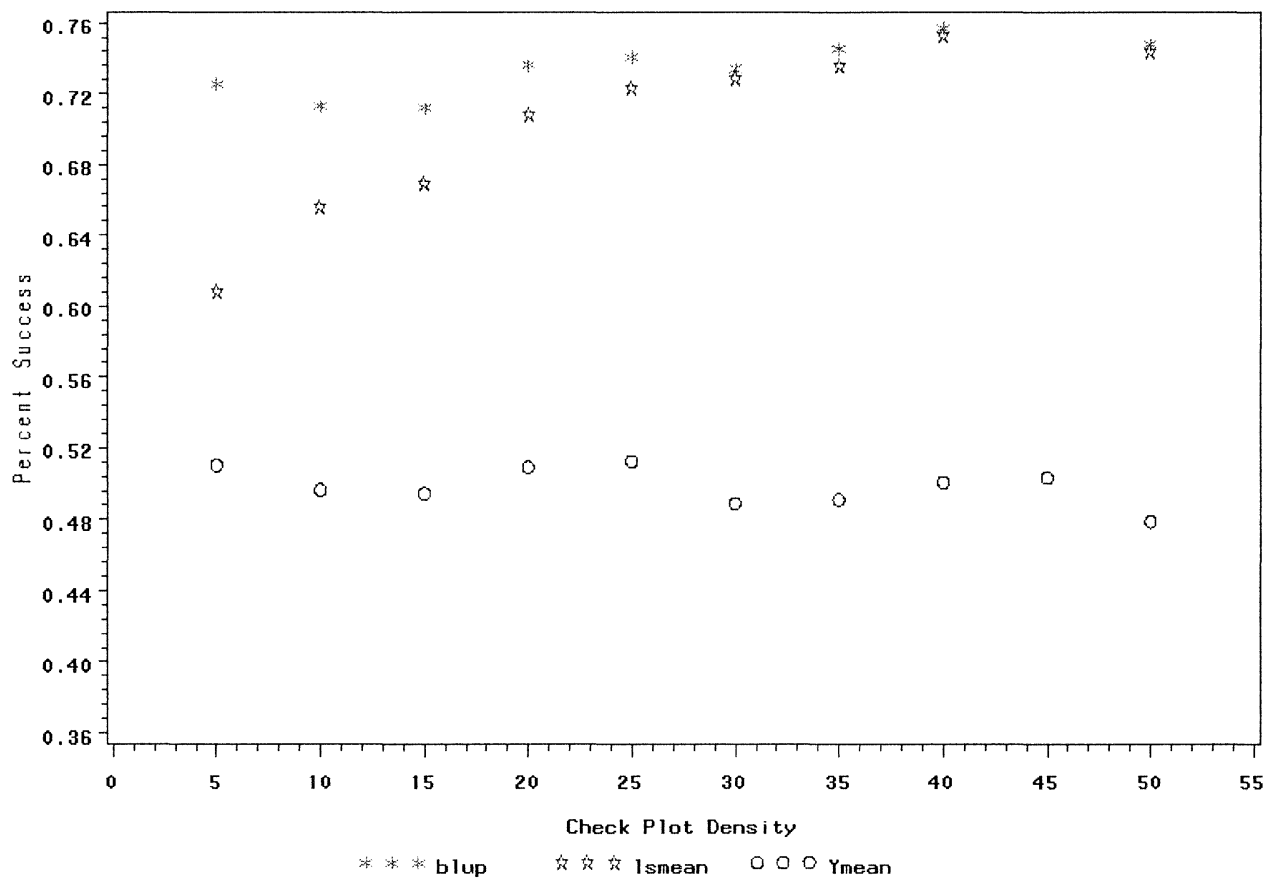

